\title{
The CBC social network
}

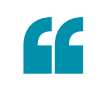

\section{human CBC subcomplexes that have specific functions in RNA} metabolism פy
The 5' 7-methylguanosine cap is a crucial element of RNA polymerase II-dependent transcripts; it protects RNAs from exonucleolytic degradation and coordinates several regulatory events (including RNA splicing, 3' end formation, turnover and subcellular localization). It is bound by the nuclear cap-binding complex (CBC), which is thought suggested to be a central node that integrates several RNA biogenesis steps. However, only a few factors have been shown to directly bind to the $\mathrm{CBC}$, and so the mechanism by which this complex routes RNAs to the correct processing pathway remains to be discovered. Two groups have now characterized human $\mathrm{CBC}$ subcomplexes that have specific functions in RNA metabolism.

Bertrand and colleagues show that the $\mathrm{CBC}$ forms a complex with arsenite-resistance 2 (ARS2), which they term CBCA. They further report that the $\mathrm{CBC}$ and $\mathrm{ARS} 2$ bind to a

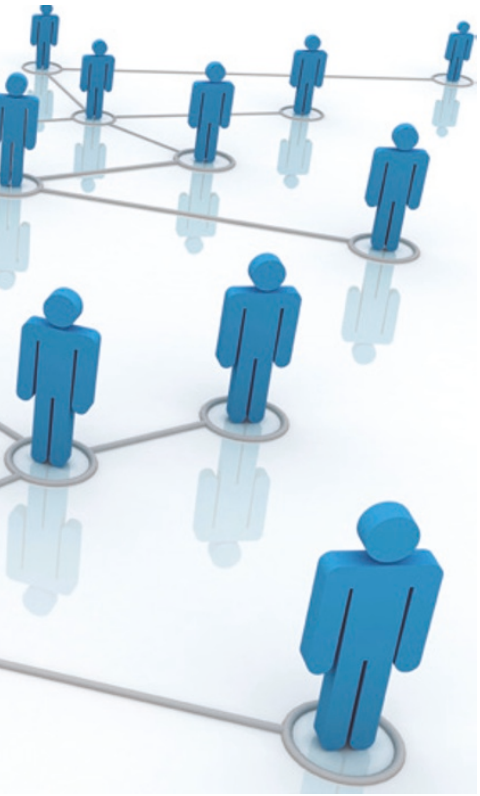

similar set of capped, non-coding and coding RNA species, and that their knockdown results in increased readthrough of $3^{\prime}$ end sites of small nuclear RNAs (snRNAs) and polyadenylated mRNAs. This suggests that ARS2 promotes 3 ' end processing in the context of the CBC. Interestingly, increasing the distance between the cap and 3 ' end processing signal decreased the read-through phenotype upon CBC and ARS2 knockdown, suggesting that CBCA promotes cap-proximal 3 ' end cleavage.

How do these factors mediate the observed effects? The authors found that the $\mathrm{CBC}$ and ARS2 associate with the cleavage factor CLP1. Testing the functional importance of this interaction, they show that CLP1 deletion also increased read-through transcription, which suggests that cap-dependent $3^{\prime}$ end processing is mediated by the association of CBCA-CLP. Finally, ARS2 binding to the $\mathrm{CBC}$ enhanced binding of phosphorylated adapter RNA export protein (PHAX; forming a complex termed CBCAP) to pre-snRNAs; thus, ARS2 seems to be a key $\mathrm{CBC}$ effector that physically and functionally couples snRNA $3^{\prime}$ end maturation and export.

Jensen and colleagues characterized a functional connection between the $\mathrm{CBC}$ and the ribonucleolytic exosome in RNA metabolism. They hypothesized that the nuclear exosome targeting (NEXT) complex, which aids in exosomal RNA degradation and which has previously been shown to bind to the CBC, bridges the cap to the exosome. They first established that a complex forms, comprising CBCA and the NEXT complex (termed CBCN). Importantly, they found that the
RNA exosome is a highly specific interaction partner of NEXT, which is bound to the CBCA complex. This suggests that the CBCA has a role in targeting RNA to the exosome. Consistent with this idea, CBCN components associated with promoter upstream transcripts (PROMPTs), which are known NEXT-exosome substrates. Next, depletion of $\mathrm{CBCN}$ components resulted in the accumulation of PROMPTs, with the deletion of the CBC component CBP80 and ARS2 causing transcriptional read-through and increased amounts of $3^{\prime}$ extended PROMPTs, which suggests that ARS2 promotes early transcription termination of this RNA class. Importantly, these read-through transcripts accumulated further in the absence of the exosome, providing evidence for a functionally relevant link between the RNA 5' cap and the ribonucleolytic activity of the exosome.

In summary, these studies suggest a model in which cap-proximal transcriptional termination, mediated by CBCA, drives co-transcriptional RNA degradation as well as a potentially important role for CBCA in discriminating between functional and cryptic transcripts. These effects require the formation of diverse CBC subcomplexes, which determine RNA fate by connecting $3^{\prime}$ end formation, degradation and export.

Andrea Du Toit

ORIGINAL RESEARCH PAPERS Hallais, M. et al. CBC-ARS2 stimulates 3'-end maturation of multiple RNA families and favors cap-proximal processing. Nature Struc. Mol. Biol. http://dx.doi. org/10.1038/nsmb.2720 (2013)| Refsing Andersen, R. et al. The human cap-binding complex is functionally connected to the nuclear RNA exosome. Nature Struc. Mol. Biol. http://dx. doi.org/10.1038/nsmb.2703 (2013) 